\title{
Hierarchical Hidden Markov Models An Application to Health Insurance Data
}

\author{
Ah Chung Tsoi ${ }^{1}$, Shu Zhang ${ }^{2}$, and Markus Hagenbuchner ${ }^{2}$ \\ 1 Australian Research Council, GPO Box 2702 Canberra, ACT 2601 \\ 2 Faculty of Informatics, University of Wollongong, NSW 2522
}

\begin{abstract}
This paper provides a constructive algorithm in which a hierarchical tree of hidden Markov models may be obtained directly from data using an unsupervised learning regime. The method is applied to health insurance transaction data such that profiles with similar local temporal behaviours are grouped together. By judicious incorporation of limited additional prior information, it is found that profiles can be separated into various sub-behavioural groups thus providing a technique for large-scale automatic labelling of data. In the application to the health insurance transaction data set, by incorporating limited information concerning the medical functions used in a medical procedure, it is possible to label some individual medical transactions as to whether they are related to a particular medical condition or not. This automatic labelling process adds values to the collected transactional database for possible further applications, e.g. public health studies.
\end{abstract}

\section{Introduction}

The study undertaken in this paper is motivated by the following common data mining problem: Given a large number of temporal profiles with unknown classifications, can we classify them into various behavioural groups? In general this problem is solvable as there are a number of unsupervised learning algorithms which can classify the data into various behavioural groups. For example, we may be given a database of credit card transaction records. It is possible to use various unsupervised learning methods, e.g. $\mathrm{K}$-mean clustering to classify the records into clusters of behavioural groups; in this case, profiles of credit card usage by their temporal behaviours over a particular period. However, often such grouping of data is of limited practical applications, as it is not possible to label the clustered data. If however in addition, we are given some labelled data then it may be possible to generalize the underlying information captured by the labelled data to the entire set of temporal profiles. Such labelled data set will be of much practical value. For instance, in the credit card example, if we are given some "ground truth" information, e.g. profiles which are known to be "fraudulent" (through forensic examination of the profiles, and successfully validated through expert opinions), then it is possible to use supervised techniques to train a model and then use the trained model to classify the entire data set into labelled data. However, often the "ground truth" data is very expensive or impossible to obtain. In the credit card example, it is known that it is expensive to obtain a small set of curated "ground truth" validated training examples. Secondly, in a supervised training regime, often for good generalization accuracy, the 
number of training data required needs to be carefully chosen, so that (a) they cover most of the common behavioural patterns which the user wishes to capture in the data set, (b) they are sufficiently varied as to cover all "eventualities" in the data set. This implies that a human is capable of choosing exemplars from a large number of data, and has sufficient domain knowledge as to know that a particular profile represents a typical behaviour which is to be captured, and secondly, knows that the selected profiles will have sufficient diversity or "richness" so that the supervised training of the model would converge to a model capable of generalizing well. Obviously the choice of suitable training exemplars from a large set of data is very much an art rather than a science.

In many practical examples, it may be too expensive to obtain a large set of training examples, or have a limited understanding of the underlying system and this prevents human experts from assessing the suitability of particular data for training purposes. This is especially true in cases where there is a large amount of data. As an example, a computer system administrator in a large organisation can capture the network traffic through say a border router. The aim may be to detect any intrusion attempts into the computer system under study. The advance of the art of intrusion detection has not progressed to such an extent that a human system administrator can look at such traffic record and determine whether a particular record is a suitable training example.

In this paper we are interested in a particular domain application which involves routinely collected and unlabelled data. More precisely, we are given detailed transactional records of a large health insurance vendor. The transactional record contain de-identified claimant details, e.g. unique personal identifier (which cannot be reverse engineered to ensure privacy), time of claim, amount of claim, and brief description of the purpose of the claim which is provided in the form of an item. The item only pertains to the medical procedure performed on the claimant by the medical service provider. It does not pertain to diagnostic information on the medical condition of the claimant. Often the same item is used for a variety of (related) ailments, thus without access to the diagnostic information of the medical service provider, it is very difficult to associate the items used with a particular course of treatment as a "proxy" indicator of the medical condition suffered. Our challenge is to label a sub-group of the large amount of such transactional records. In particular, the question which we wish to answer is: can we label some transactional records as representing cases featuring particular ailments. It is obvious that it will not be possible to label all transactional records into unique subgroups, say, claimants suffering from the common cold, as there is simply insufficient information to allow us to do so. On the other hand, some items are specific to particular ailments, e.g. stroke sufferers often are provided with treatments represented by item numbers $63130,63218,63850^{3}$. The question becomes: can we label sub-groups of medical transaction records using such item numbers. A naive answer would be: search all medical transaction records for all occurrences of such item numbers. However, an indication of a stroke sufferer is someone who uses one or all of these items as well as a large number of other items. In other words, even if a person uses item 62130, the person might not be suffering from a stroke. The item is used if an investigation is

\footnotetext{
${ }^{3}$ These items are related to magnetic resonance imaging scans or magnetic resonance angiography. They relate to investigation of the occurrence of stroke in the claimants, or the monitoring of claimants who have been diagnosed as suffering from strokes.
} 
being carried out to ascertain if the claimant might be suffering from a stroke. Secondly the medical service providers may use many other items to determine if the person is suffering from a stroke or not. Hence by searching through the database for occurrence of these records may not capture all possible sufferers of the illness. The question is that there needs to be a way in which we can with some confidence indicate that a person is suffering from strokes. A fundamental assumption underpinning our work is: claimants suffering from the same ailments would have similar temporal behaviours as exhibited by their Medicare claim behaviours over a period of time. This assumption is demonstrated to be valid ex poste from our results.

The value to the data set is enormous as if we can overcome this challenge, as it would be possible to automatically label sub-groups of these transactional records into various sub-groups as sufferers of diabetes, stroke, etc. Once labelled as such, the database may be used for other purposes, e.g. the geographical distribution of stroke sufferers in the country, at a particular moment in time, and its variation over a period of time. In combination with data from other sources, e.g. general dietary information from supermarkets, amount of exercise of small scaled surveys, it may be possible to derive some general conclusions on the cause and effect of stroke illness.

In this paper, we will only consider the first part, i.e. how to label a sub-group of transactional records as claimants suffering from various illnesses. We will not be concerned with the second part, viz., its possible public health applications once such labelling can be performed.

Our data consists of $180 \mathrm{~GB}$ of de-identified transactional records which covers seven consecutive quarters of transactions ${ }^{4}$. Our task is: label a sub-group of such records into claimants suffering from diabetes, stroke, etc.

Faced with such task, we proceed as follows:

(1) Feature extraction to extract representative features of the underlying temporal behavior of the claimant. In our case, we extracted the following information: unique personal identifier, date of claim, age of the claimant, total claims per day.

(2) Creation of cohorts considers the fact that a claimant's medical records change dramatically with age. Claimants of similar age range form an age cohort. Steps (1) and (2) are pre-processing steps which prepare the data for the application of pattern discovery techniques.

(3) Clustering. Gaussian mixture clustering is applied to identify clusters in the data for each age cohort. This step gathers profiles together according to an Euclidean norm based on the entire temporal profile, rather than based on temporal variations within the profile.

(4) Pattern discovery is accomplished by using hidden Markov models (HMM), a stochastic model commonly used in temporal behavioral pattern discovery ${ }^{5}$. Steps

\footnotetext{
${ }^{4}$ This data set has been provided by the Health Insurance Commission (HIC), Australia, a national medical insurance vendor which provides universal health cover to all Australian citizens and permanent residents.

${ }^{5}$ HMMs are not particularly suited for large scale data mining tasks given the high computational demand associated with the training of these models. This problem is alleviated through the recursive training of HMMs on small portions of the data set; a step which will be addressed in greater detail later in this paper.
} 
(3) and (4) are executed recursively until convergence occurred. This recursive process yields a set of HMMs which are hierarchically organised.

(5) Automatic annotation. Without any additional information, the clusters from recursive applications of steps (3) and (4) will contain temporal behaviours of claimants who might be suffering from various ailments. In other words, different ailments will produce similar temporal behavioral patterns. In order to disambiguate these similar patterns, additional information on the medical treatments received by claimants in the form of items used by the medical service providers is used to further separate the patterns within the same cluster as obtained in steps (3) and (4) into ailments which the claimant might be suffering ${ }^{6}$. Thus, by utilizing the information on medical items used by the medical service providers, we are able to obtain an automatic annotation technique which can annotate large scale medical transaction records.

The organization of this paper is as follows: Section 2 describes the techniques used in the pre-processing steps. A general discussion of pattern discovery is given in Section 3. Our method will be presented in Section 4. Experimental results are shown in Section 5. Conclusions are drawn in Section 6.

\section{Representation of temporal sequences}

As indicated in Section 1, we will use the total claim per day from a claimant, the date when the service was rendered, and the age of the claimant to construct a profile. In addition, we smooth out the fluctuations (as not everybody sees a medical service provider every day) by using a sliding window of 14 days to compute the total claim made over that period. Ex poste this was found to be appropriate as a sick person is often required to see a medical service provider a number of times over a short period. We use an annual cycle of 365 days, and account for boundary effects as benefit claims often are not lodged on the same day as they are rendered. This results in temporal profiles such as those shown in Figure 1.

A claimant's medical condition can change dramatically with age. We analysed the data and observed that there are abrupt changes between certain age groups. Consequently, we segmented the profiles into age cohorts as shown in Table 1. Segmenting the profiles into cohorts of claimants of similar age gives two advantages: First, it reduces the size of the dataset for later analysis tasks, and secondly it reduces the impact of age related medical issues on the learning tasks.

In Table 1 we find that for both male and female claimants the amount of benefit paid and the frequency of medical services decrease until the claimants reach adolescence. Then these values experience a rapid increase before slowing down until retirement age for female claimants; a similar trend can be observed for male claimants but at a much slower rate.

In summary, a dimension reduction of the dataset is achieved by setting up a onedimensional profile for each claimant, and the dataset is segmented into age cohorts. The pattern discovery techniques will be applied to each age cohort separately.

\footnotetext{
${ }^{6}$ Note that we do not have access to medical service providers' diagnosis nor notes.
} 


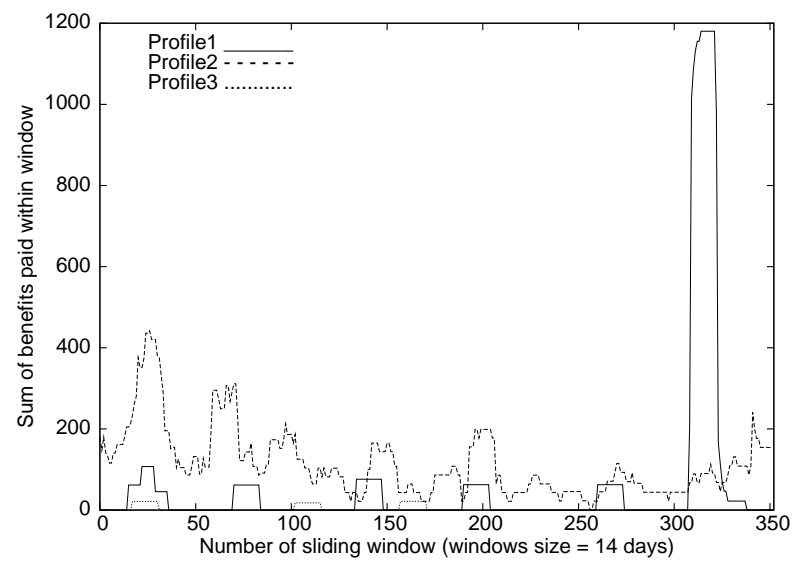

Fig. 1. Three profiles from the dataset.

Table 1. Cohort groups and sizes.

\begin{tabular}{|c|c|c|c|c|c|c|}
\hline & \multicolumn{3}{|c|}{ Female } & \multicolumn{3}{|c|}{ Male } \\
\hline $\begin{array}{l}\text { Age } \\
\text { cohor }\end{array}$ & & & & & & $y=y$ \\
\hline $0-3$ & 460,548 & 7.65 & 229.94 & 489,403 & 8.29 & 257.56 \\
\hline $4-6$ & & & & & 5.7 & \\
\hline $7-15$ & 957, & 4. & & 99 & 4.3 & .58 \\
\hline $16-24$ & $1,053,985$ & 7.41 & & 946,464 & 4.77 & 196.22 \\
\hline $5-35$ & $1,498,662$ & 9.0 & 8 & 1,257 & 5.2 & .97 \\
\hline $6-44$ & 1 & 8.5 & 4 & 17 & 6.0 & 41 \\
\hline $5-55$ & 334 & 9.8 & & 1,19 & 7.3 & \\
\hline $56-70$ & $1,101,022$ & 12.73 & 684.27 & $1,068,311$ & 11.21 & 654.19 \\
\hline$>70$ & 862,858 & 16.97 & 875.29 & 556,972 & 15.07 & 715.07 \\
\hline
\end{tabular}

\section{Pattern discovery techniques}

This section gives a general introduction to Gaussian Mixture models and hidden Markov models which will be employed in the pattern discovery task later in this paper. We will also describe a method to obtaining a hierarchical tree of hidden Markov models from the set of claimant profiles. This is a two step process:

Step 1 Clustering of the entire profiles. In this step, we will group profiles together according to their overall pattern. This is achieved by using a clustering technique such as a Gaussian mixture model.

Step 2 Use a hidden Markov model to further divide the clusters obtained in Step 1 into clusters of similar temporal behavioral patterns.

\subsection{Gaussian mixture model as a clustering tool}

The task of discovering temporal patterns could be initialized by clustering because of its ability to segment data into clusters according to a similarity criterion. There 
are a number of possible algorithms which can be employed for this purpose, e.g., K-means clustering algorithm [4], Gaussian mixture algorithm [5], mixture of HMMs [11], self organizing map method [6]. Mixture of HMMs and Self-Organizing Maps are computationally expensive to be used given the size of the dataset. The K-means algorithm has been tried but the results obtained indicated that "sparse" vectors are not clustered well. Sparse vectors are common occurrences in the given dataset as most claimants do not visit a medical service provider frequently. Consequently, we did not use the K-means algorithm in any further experiments. In this respect, Gaussian mixture (GM) models are more practical in terms of computational demands [3].

With GM, it is assumed that the data observed are generated by a $\mathcal{D}$-dimensional GM of $N$ components ( $N$ clusters) with the following probability density function [3]: $f(\mathbf{x} \mid \Psi)=\sum_{n=0}^{N-1} p_{n} g\left(\mathbf{x} \mid \Theta_{n}\right)$ where $\Psi$ denotes the vector encompassing all the mixture parameters $\left(\Theta_{n}\right.$ and $\left.p_{n}\right), p_{n}\left(\geqslant 0, n=0,1,2, \ldots, \mathrm{N}-1, \sum_{n=0}^{N-1} p_{n}=1\right)$ is the weight of the $n-t h$ component in the model, or the possibility that the pattern $\mathbf{x}$ was generated from the $n-t h$ component. $\Theta_{n}$ stands for all the parameters $\left(\boldsymbol{\mu}_{n}\right.$ and $\left.\boldsymbol{V}_{n}\right)$ of the $n-t h$ $\mathcal{D}$-variate Gaussian distribution $g\left(\mathbf{x} \mid \Theta_{n}\right)$ of probability density function: $g\left(\mathbf{x} \mid \Theta_{n}\right)=$ $\frac{\exp ^{-\frac{1}{2}(\mathbf{x}-\boldsymbol{\mu})^{T}(\boldsymbol{V})^{-1}(\mathbf{x}-\boldsymbol{\mu})}}{\sqrt{(2 \pi)^{D} \operatorname{det}\left(\boldsymbol{V}_{n}\right)}}$ with mean vector $\boldsymbol{\mu}_{n}$ and covariance matrix $\boldsymbol{V}_{n}$.

In general, the parameters of the model are typically trained using expectation maximization (EM) algorithm [3] which is used to produce the maximum likelihood estimates $\hat{\boldsymbol{\mu}}_{n}$ and $\hat{\mathbf{V}}_{n}$ to parameters $\boldsymbol{\mu}_{n}$ and $\boldsymbol{V}_{n}$, respectively. More importantly, the parameters can be improved through some EM iterations [7] with respect to the dataset $\mathbf{x}=\left\{\mathbf{x}_{m}, \mathrm{~m}=0,1, \ldots, \mathrm{M}-1\right\}$ so that the model will fit the data better. The updating of parameters in the $k$-th EM iteration is as follows [7]:

$$
\begin{array}{lc}
h_{n}^{(k)}\left(\mathbf{x}_{m}\right)=\frac{p_{n}^{(k-1)}\left(\mathbf{x}_{m}\right) g\left(\mathbf{x}_{m}, \Theta_{n}^{(k-1)}\right)}{f^{k-1}\left(\mathbf{x}_{m}\right)} & \hat{p}_{n}^{(k)}(\mathbf{x})=\frac{\sum_{m=0}^{M-1} h_{n}^{(k)}\left(\mathbf{x}_{m}\right)}{M} \\
\hat{\boldsymbol{\mu}}_{n}^{(k)}(\mathbf{x})=\frac{\sum_{m=0}^{M-1} \mathbf{x}_{m} h_{n}^{(k)}\left(\mathbf{x}_{m}\right)}{M \hat{p}_{n}^{(k)}(\mathbf{x})} & \hat{\mathbf{V}}_{n}^{(k)}(\mathbf{x})=\frac{\sum\left(\mathbf{x}_{m}-\hat{\boldsymbol{\mu}}_{n}^{(k)}(\mathbf{x})\right) h_{n}^{(k)}\left(\mathbf{x}_{m}\right)\left(\mathbf{x}_{m}-\hat{\boldsymbol{\mu}}_{n}^{(k)}(\mathbf{x})\right)^{T}}{M \hat{p}_{n}^{(k)}(\mathbf{x})}
\end{array}
$$

where $\hat{p}_{n}^{(k)}(\mathbf{x}), \hat{\boldsymbol{\mu}}_{n}^{(k)}(\mathbf{x})$ and $\hat{V}_{n}^{(k)}(\mathbf{x})$ are the maximum likelihood (ML) estimators for the unknown parameters $p_{n}, \boldsymbol{\mu}_{n}$ and $\boldsymbol{V}_{n}$.

The EM iteration will stop when one of the following criteria is satisfied: (i) not enough improvement, $1 \leq \frac{L\left(\hat{\Psi}^{(k)}\right)}{L\left(\hat{\Psi}^{(k-1)}\right)} \leq 1+\gamma$, where $\gamma>0$ is given by user, and $L\left(\hat{\Psi}^{(k)}\right)=\sum_{m=0}^{M-1} \log \left[\sum_{n=0}^{N-1} \hat{p}_{n}^{(k)} g\left(\mathbf{x}_{m} \mid \hat{\Theta}_{n}^{(k)}\right)\right]$, or (ii) $k=K, K$ is the maximum number of EM iterations.

After the updating of parameters through the EM algorithm, a Gaussian mixture model is able to group each $\mathbf{x}_{m}(\mathrm{~m}=0,1,2, \ldots, \mathrm{M}-1)$ into a cluster $c$ if $\beta_{n}(\mathbf{x})=$ $\log \left[\hat{p}_{n} g(\mathbf{x} \mid \hat{\Theta})\right]$ and $c=\arg \max _{n=0,1,2, \ldots, N-1} \beta_{n}(\mathbf{x})$.

With the GM model, a problematic issue is the choice of the number of components $N$. The number of components could be assigned based on some prior knowledge of the dataset, or obtained by applying some information criteria (IC) [8,9], which provides a rational choice of the number of components based on Bayesian or similar arguments. In our work, we chose the number to be 6 through some preliminary experiments on the dataset while IC will be considered in future work. 


\subsection{The Hidden Markov Model}

A hidden Markov model (HMM) is a system of states with a probabilistic state transition model which can model a given sequence of events. A number of variants of HMMs are available [10], such as discrete HMM, continuous observation HMM, input-output HMM. In our case, a continuous HMM is deployed. It is assumed that the observations $\mathbf{y}_{1}, \mathbf{y}_{2}, \ldots, \mathbf{y}_{T}$ are generated by a multivariate probability density function. For simplicity, we will assume that this is generated by a Gaussian mixture as follows:

$$
f_{\mathbf{y} \mid \mathbf{x}}(\xi \mid i)=\sum_{m=1}^{P} c_{i m} \mathcal{N}\left(\xi ; \mu_{i m}, C_{i m}\right)
$$

where $\mathcal{N}\left(\xi ; \mu_{i m}, C_{i m}\right)$ denotes a Gaussian probability density function with mean $\mu_{i m}$ and covariance matrix $C_{i m}$. The notation $f_{\mathbf{y} \mid \mathbf{x}}(\cdot)$ denotes the probability of observing $\mathbf{y}$ given the hidden state sequence $\mathbf{x}$. The constants $c_{i m}$ are known as mixing coefficients. In order to be a probability density function, we must have $\sum_{m=1}^{P} c_{i m}=1$ for $1 \leq i \leq$ $S$, and $S$ is the size of the alphabet (the dimension of the state space).

It is further assumed that the observation probability density functions are generated by a hidden state $\mathrm{x}, \mathrm{x}$ is a $S$ dimensional vector which follows the evolution equation:

$$
\mathbf{x}(t+1)=A \mathbf{x}(t)
$$

where $A$ is the state transition matrix, with initial condition $\mathbf{x}(0)=\pi_{0}$. The parameters in the model are then $\mathcal{M}=\left\{S, \pi_{0}, A,\left\{f_{\mathbf{y} \mid \mathbf{x}}(\xi \mid i), 1 \leq i \leq S\right\}\right\}$.

The problem in HMM estimation can be divided into two sub-problems: (1) given a series of training observations for a given entity, say, a label, how do we train a HMM to represent this label? This problem becomes the finding of a procedure for estimating an appropriate state transition matrix $A$, and observation probability density function $f_{\mathbf{y} \mid \mathbf{x}}$ for each state. (2) given a trained HMM, how do we find the likelihood that it produced the incoming observation sequence.

The HMM estimation algorithm is readily available in e.g., $[1,2,10]$. We will use the training algorithm presented in [10]. For the problem of finding class labels given a set of observations, the Viterbi algorithm [10] is used.

\section{The proposed method}

Our approach to the task of pattern discovery is presented in this section. A two-step approach is used which is then applied recursively to obtain a hierarchical tree of HMMs. In the first step, GM is engaged to detect clusters among the profiles. The second step trains a set of HMMs, one for each cluster of profiles. To reduce the computational burden, the HMMs are trained recursively on relatively small subsets. In each iteration, the HMMs are trained on a different subset of randomly selected profiles from the cluster until the classification error reaches a minimum. Note that this approach to training is similar to the k-fold cross validation approach [12], except that it is not as formal. In our approach, we do not divide the data formally into k equal groups. Instead we divide the data into two groups, which may not be equal in size. Then, we randomly select the 
profiles in one group for training. The evaluation is performed on other groups of data not used in the training data set. Then the data set is further refined using the HMM. The number of profiles in each group may be different. We do not report the average generalisation error. Instead we report the error obtained on a particular set of testing data set. The use of k-fold cross validation process may improve our proposed methodology, as it provides a formal method for training and evaluating data. Furthermore some convergence results have been proved [12]. This will be an issue for further research as it will be interesting to see how different would the results be using our ad hoc method compared with the more formal cross validation methods [12].

\subsection{Gaussian mixture clustering}

It was mentioned in Section 2 that cohorts of claimants of similar age are considered for the pattern recognition task. In this section we choose age cohort 56-70 to illustrate the approach. There are a total of 2,169,333 profiles in this age cohort; we will divide them into a training data set and a validation data set. The GM clustering approach addressed in Section 3.1 is employed on 91,219 randomly selected profiles from the data pool. We employ this relatively small subset of data rather than the full data set available in this cohort group as it suffices for illustration purposes and it allows us to reduce the turn around time for experiments. The subset was obtained by selecting all claimant profiles from a number of postcode areas. Special care was taken to ensure that patients from rural areas and metropolitan areas are proportional to the entire dataset. The list of postcode areas was hand-picked. Postcode areas were obtained by looking up on a geographical map. It was then decided to whether or not to include this postcode area in for the subset. This procedure was tailored to reduce the risk of possible side affects which may arise out of randomly choosing patient profiles. Since both, GM and HMM scale linearly with the number of training data, the training time required for the full dataset is easily estimated through a linear adjustment of the training times stated in this paper. It is important to note that the size of this subset has been chosen so that it contains a good representation of the features available in the full data set.

Out of 91,219 profiles, 64, 553 are used for the training process; all remaining profiles are used for testing purposes. Again, the size of the training set is influenced by a proportional selection of rural and metropolitan postcode areas. All claimants whose residential addresses that fall within the selected postcode areas are selected (not just some of them). We assume that the dataset is generated by a Gaussian mixture model of $N=6$ components ${ }^{7}$. The parameters of the model will be first estimated by utilizing the EM algorithm, and then updated through a few EM iterations as shown in Section 3.1 for a better representation to the dataset. The updating ends when improvement of the log likelihood of the posterior probability is less than $1 \%$ or the number of EM iteration reaches 50. We applied the GM algorithm to segment the 64,553 profiles into 6 clusters as shown in Table 2.

These clustering results are not suitable for the task of temporal pattern discovery in the sense that it only provides a clustering based on the entire profile using say an

\footnotetext{
${ }^{7}$ We had tried a number of values for $N$ and found that $N=6$ produces well behaving hierarchical trees of HMMs as is shown later in this paper.
} 
Table 2. The clustering results of Gaussian mixture model with 6 components.

\begin{tabular}{|l|c|c|c|c|c|c|c|}
\hline Cluster: & A & B & C & D & E & F & Total: \\
\hline Size: & 10780 & 10225 & 17762 & 11105 & 10315 & 4366 & 64553 \\
\hline
\end{tabular}

Table 3. Classification results by the hidden Markov model.

\begin{tabular}{|c|r|r|r|r|r|r|r|}
\hline Class & A & B & \multicolumn{1}{c|}{ C } & \multicolumn{1}{c|}{ D } & E & F & Total \\
\hline A & $\mathbf{9 4 8 5}$ & 1285 & 10 & 0 & 0 & 0 & 10780 \\
\hline B & 4055 & $\mathbf{3 5 1 6}$ & 2224 & 430 & 0 & 0 & 10225 \\
\hline C & 957 & 7002 & $\mathbf{9 1 4 6}$ & 657 & 0 & 0 & 17762 \\
\hline D & 0 & 0 & 2019 & $\mathbf{5 7 0 1}$ & 2787 & 598 & 11105 \\
\hline $\mathrm{E}$ & 0 & 0 & 186 & 6207 & $\mathbf{3 2 8 2}$ & 640 & 10315 \\
\hline $\mathrm{F}$ & 0 & 0 & 0 & 0 & 879 & $\mathbf{3 4 8 7}$ & 4366 \\
\hline$\Sigma$ & 14497 & 11803 & 13585 & 12995 & 6948 & 4725 & 64553 \\
\hline
\end{tabular}

Euclidean norm. Thus, two profiles with different temporal differences but similar Euclidean norm would be grouped together in the same cluster. The coarse segmentation will be refined through the application of HMMs.

\subsection{Recursive HMM modeling}

As a widely used pattern discovery technique, HMMs are capable of classifying profiles with similar temporal patterns into the same class. The clustering results of GM could serve as training sets so that $6 \mathrm{HMMs}$, one for each cluster, are trained. However, the computational efforts of training hidden Markov models render them not particularly suitable for data mining tasks, e.g., it takes about 250 minutes to train a HMM on 10,780 profiles using a workstation with a $2 \mathrm{GHz}$ XEON processor, and 2 GB RAM. We propose to take a recursive training-recognizing cycle on subsets of data to ease this computational burden:

Training phase: No more than 2000 profiles are randomly chosen from each cluster to train a corresponding HMM. By doing so it takes about 13 minutes to train a HMM.

Recognition phase: All 64,553 profiles are classified by the trained HMMs. The classification re-distributes the profiles between the 6 clusters as shown in Table 3. The rows in Table 3 refer to the clusters obtained by GM whereas the columns are the classifications produced by the set of HMMs. The result will be used for further processing.

The off-diagonal numbers of the confusion Table 3 describe how GM segments different profiles from the HMM. For example, the number of profiles in Class $\mathrm{E}$ fell from 10,315 to 6,948 . Thus, the recognizing phase provides an adjustment to the grouping of profiles by considering temporal patterns discovered in the training set.

The result may not be optimal since only a relatively small number of profiles were engaged during the training process. The quality of HMMs is improved through a recursive application of training-recognition cycle by adopting the grouping results from the recognition phase of the previous cycle. This iterative process ensures that more and more data from the training set is eventually considered in the training process, and that mis-classifications are reduced. 
Table 4. Classification results by the trained HMMs when reaching the stopping criteria.

\begin{tabular}{|c|r|r|r|r|r|r||r|}
\hline Class & \multicolumn{1}{|c|}{ A } & \multicolumn{1}{|c|}{ B } & \multicolumn{1}{c|}{ C } & \multicolumn{1}{c|}{ D } & E & F & Total \\
\hline $\mathrm{A}$ & $\mathbf{9 0 3 0}$ & 0 & 0 & 0 & 0 & 0 & 9030 \\
\hline $\mathrm{B}$ & 14 & $\mathbf{1 4 2 2 7}$ & 11 & 0 & 0 & 0 & 14252 \\
\hline $\mathrm{C}$ & 0 & 1 & $\mathbf{1 6 4 5 7}$ & 0 & 0 & 0 & 16458 \\
\hline $\mathrm{D}$ & 0 & 0 & 64 & $\mathbf{1 3 9 5 2}$ & 0 & 0 & 14016 \\
\hline $\mathrm{E}$ & 0 & 0 & 0 & 8 & $\mathbf{8 8 5 3}$ & 0 & 8861 \\
\hline $\mathrm{F}$ & 0 & 0 & 0 & 0 & 3 & $\mathbf{1 9 3 3}$ & 1936 \\
\hline$\Sigma$ & 9044 & 14228 & 16532 & 13952 & 8856 & 1933 & 64553 \\
\hline
\end{tabular}

The recursive application of training-recognizing cycle stops when there are no misclassifications or when a maximum number of iterations is reached. Here we allow it to run for up to 50 cycles. The resulting confusion matrix as shown in Table 4 shows that the classification of profiles is vastly improved.

In summary, the recursive training of HMMs significantly reduces the computational burden since it provides a mechanism to detect redundancy in the data set. This is due to the fact that mis-classifications will approach zero faster if the dataset contains many redundant data.

\subsection{Building a hierarchical tree of HMMs}

In the previous 2 steps of GM-HMM pattern discovery process, we have successfully trained HMMs as a representation of patterns. Here we show how the quality of the HMM representations can be further enhanced. At the 40-th cycle it is observed that this is still a gross segmentation of the set of profiles since it is impossible to be certain about the number of clusters which can be used to describe the dataset. The refinement of classification starts with another GM-HMM pattern discovery process for each of the classes obtained so far unless the size of a class is too small to proceed, say, less than 300 profiles $^{8}$. Figure 2 explains the process in a visual manner. The first iteration of the GM-HMM process at the top level ends up with 6 classes after 50 iterations of training of HMMs which reaches the best performance at the 40-th cycle. At the 40-th cycle, another iteration of GM-HMM process at sub-level 1 has proceeded to each of the classes (A to F) for further refinement of the classification results. It is noted that instead of 50, there are only 40 iterations of HMMs training conducted in Class A, i.e., the HMMs trained at the 39-th and the 40-th cycles respectively classify the profiles of Class A in the same manner therefore the HMM training stops. The early exit of HMM training indicates that the HMMs trained on the 2000 randomly chosen profiles from the classes are robust especially when the size of the data set is relatively large. A similar observation can be made on the GM-HMM process to Class F, where only 14 iterations have been run before the mis-classification to the profiles converges to zero. Another observation is that the GM algorithm only provides 4 classes to the profiles in

\footnotetext{
${ }^{8}$ We set 300 as the threshold since we find that a HMM cannot be properly trained on less than 300 training data. No HMM will be trained on clusters that contain less than 300 profiles. Affected profiles are considered in the recognition phase to ensure that no profile is discarded.
} 


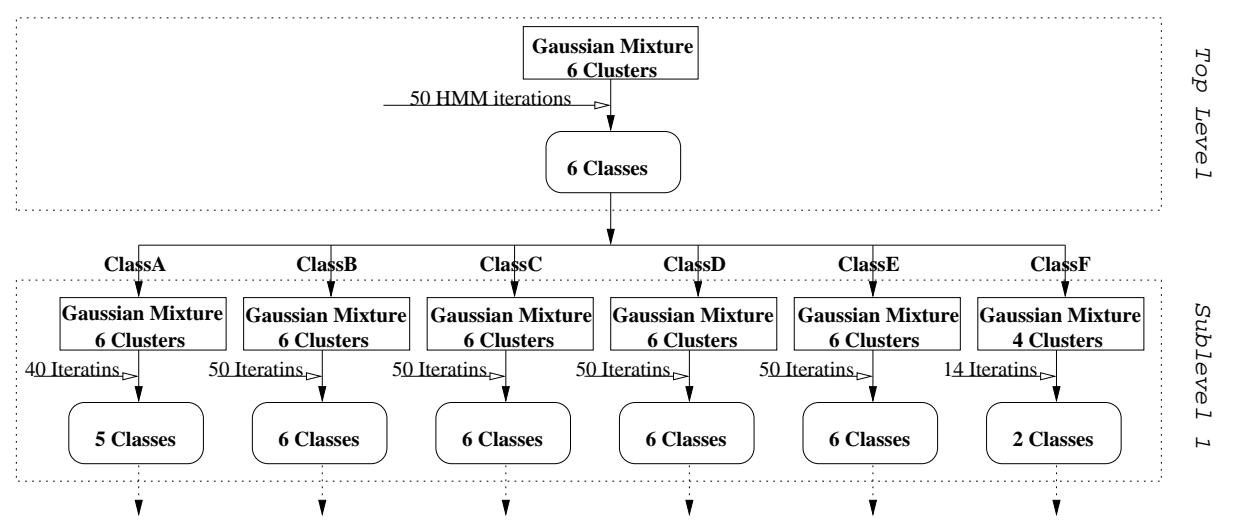

Fig. 2. A flowchart of the iterative GM-HMM refinement process.

Class F rather than the default number of 6. This implies that the GM algorithm is able to reduce the number of clusters, given the default value. As it is pointed out in Section 4.2, the quality of HMMs can be improved through the recursive deployment of training and recognition cycles to the profiles until the stopping criterion is reached. Then, yet another GM-HMM process is about to start to further refine the classifications when the mis-classification reaches a minimum as long as the size of class is large enough. Figure 3 uses Class A to provide a detailed example of the tree-like recursive application of the GM-HMM process

Through this refinement process, we decompose the bigger classes into smaller ones so that each HMM is more specialized on the patterns it represents. Our tree-like approach to pattern discovery ends up with 76 classes ( $A_{1}$ to $A_{10}, B_{1}$ to $B_{17}, C_{1}$ to $C_{20}$, $D_{1}$ to $D_{16}, E_{1}$ to $E_{10}$ and $F_{1}$ to $F_{3}$ ). The average benefit paid for each claimant in a class provides a way for us to 'name' clusters: the higher the average value, the higher the class order. e.g., the average benefit paid for claimants in class $B_{1}$ is lower than that of class $B_{2}$. But it does not guarantee that the value of a subclass from class A, say $A_{10}$, is lower than that of a subclass of $\mathrm{B}$, say, subclass $B_{1}$. The alphabetical order of the classes is decided by the maximum average value of all its subclasses.

\section{Experimental results}

Note that the profiles are unlabeled, and the HMMs were trained in an unsupervised fashion. In order to give a meaning to the classes and patterns represented by HMMs, and in order to assess the quality of the results we extract properties on the claimants in the training set and determine how these claimants are classified. For example, it is interesting to see how claimants suffering from a given illness are distributed across the classes. It may be expected that claimants suffering from similar ailments are classified into the same class. This arises from the assumption that the treatment of a particular ailment requires a particular course of treatment, and hence, a "signature" pattern may be present within a profile which can help to classify claimants with similar medical condition into the same class. 


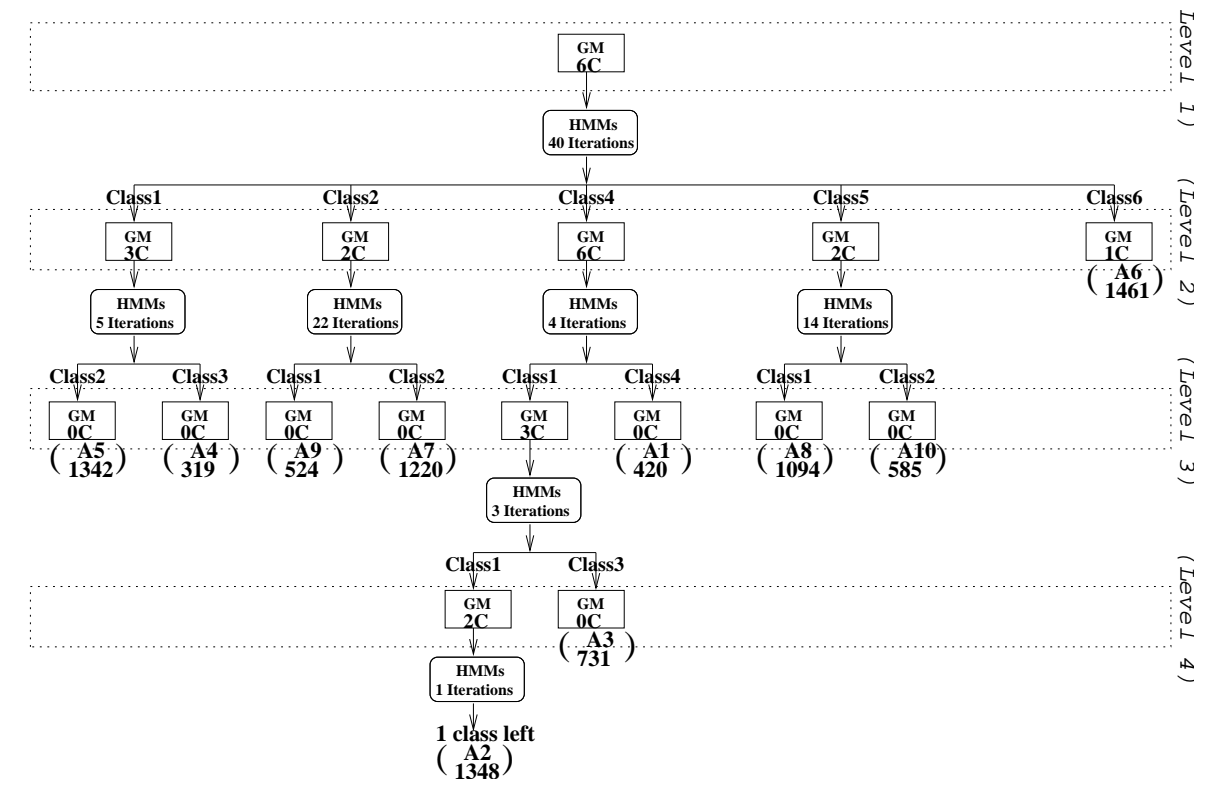

Fig. 3. Applying GM-HMM iteratively to profiles in Class A. The values in the brackets give the class label, and the number of profiles in the sub-class.

We found that this assumption is true ex poste, and that the proposed method works particularly well in this respect. In this paper, we will demonstrate this by considering a given ailment, say, stroke, and investigate how the hierarchical HMM models classify affected profiles. Medical items 63130, 63218, and 63850 specifically refer to investigation of whether the claimant is suffering from stroke. There were no other medical items containing the keyword "stroke" when we performed a keyword search on the Medicare Benefits Schedule ${ }^{9}$. Claimants claiming one or more of these items were classified as presented in Figure 4. It is observed that affected profiles are classified into a relatively small set of classes. We also found that for other cohort groups that cases of stroke are rarely observed, and hence were omitted in Figure 4. A closer investigation into the classification for age cohort group $>71$ shows that only five classes collect such profiles. As is observed, profiles classified as E7 are most likely to feature the property "stroke", while the nearby class E8 did not capture any such claimants.

Creating a histogram of item usages for those two classes, and then subtracting the usage count from each class resulted in a histogram shown in Figure 5. Shown are the differences in item usage between the two classes, where a line pointing in the negative direction indicates that the associated item was used more frequently by claimants in class E8, otherwise, if the line points in the positive direction then the associated item was used more frequently by claimants in class E7. The longer the line, the greater

\footnotetext{
${ }^{9}$ Note that this does not mean that there are no other items which are related to stroke management. It merely indicates that no other item description contained this specific keyword.
} 


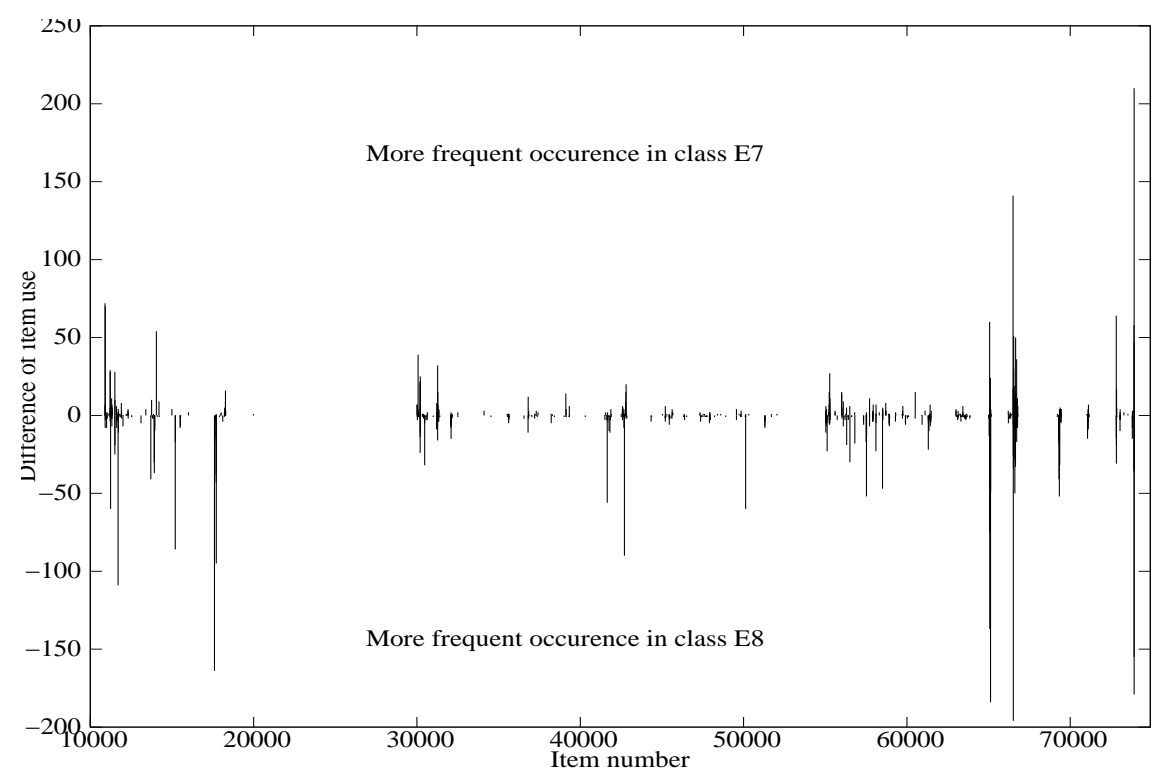

Fig. 5. Difference of item usage for claimants in class E7 and class E8.

This is a particularly interesting finding as it is shown that the proposed method introduced in this paper segments profiles into behavioural groups where each group represents a given ailment, property, or course of treatment. The example demonstrated that by judicious incorporation of limited prior information, it is possible to effectively label profiles. In this example, we have shown that class E7 indicates a likelihood that claimants classified in E7 suffer from a stroke related illness. This was confirmed by finding that many of the other profiles from the same class indicate stroke related (e.g. cholesterol studies) treatments even though a claimant has never claimed an item specifically addressing "stroke". In contrast, there is no confusion with claimants from other classes since it was shown that classes unrelated to stroke detection or stroke treatment address ailments completely unrelated to stroke. Hence, it is demonstrated that the proposed method generalizes, and discriminates well.

We also note that more than a single class is typically activated when classifying claimants with a known ailment. This is due to the fact that some ailments may not be isolated instances but are rather embedded courses of treatments within other "signatures" indicating another ailment. For example, there are claimants who receive treatment for a stroke, and at the same time receive treatment for another illness such as diabetes. Thus, some classes collect claimants who suffer from a combination of ailments.

\section{Conclusions}

In general, hidden Markov models are not particularly suitable for data mining applications as the computational loads are high when the training datasets are large. We 
have developed an effective method to overcome this limitation. Our approach first decomposes the dataset into groups of claimants of similar age since the age contributes significantly to a claimant's medical conditions. Then the approach applies recursively the Gaussian mixture clustering and HMM procedures on randomly selected samples from the training set until the classification errors converge to a prescribed minimum threshold is observed. This method is effective in detecting redundancies in the dataset and hence can contribute significantly to the reduction of the computational effort of the HMMs. Secondly, by using some limited prior information we obtain a model which is suitable for large scale automatic labeling of profiles. It was demonstrated that the model generalizes well, and is capable of assessing whether medical transactions refer to a related medical condition. This is an interesting attribute given that the model is trained unsupervised, and the underlying medical condition is not used as an input during training. The approach provides a fertile area for further studies and applications such as public health studies which can include prediction of developing ailments, geographic distribution of specific ailments, and many more.

\section{Acknowledgement}

The second and third authors wish to acknowledge financial support provided by the Australian Research Council through a SPIRT grant.

\section{References}

1. Juang, B. H., Levenson, S. E., Sondhi, M. M. "Maximum likelihood estimation for multivariate mixture observations of Markov chains", IEEE Trans on Information Theory. Vol 32, pp 307-309, 1986.

2. Liporace, L. A., "Maximum likelihood estimation for multivariate observations of Markov sources". IEEE Trans on Information Theory, Vol 28, pp 729-734, 1982.

3. McLachlan, G. J., Peel, D. "Finite Mixture Models", Wiley New York, 2000.

4. Duda, R.O., Hart, P.E., "Pattern recognition and scene analysis", J Wiley: New York, 1972.

5. Banfield, J. D., Raftery, A. E. "Model-based Gaussian and non-Gaussian clustering", Biometrics Vol 49, pp 803-821, 1993.

6. Kohonen, T., "Self-Organizing Maps", Springer, Second Extended Edition 1997, 1995.

7. J.J.Verbeek, N.Vlassis, B. Krose. "Efficient Greedy Learning of Gaussian Mixture Model", Neural Computation Vol 15, Issue 2, pp 469-485, 2003

8. Bierens, H. J. "Information criteria", http://econ.la.psu.edu/ hbierens/INFCRIT.PDF, November 2004.

9. Hastie, T., Tibshirani, R., Friedman, J. "The Effective Number of Parameters". The Elements of Statistical Learning, Data Mining, Inference and Prediction, Springer Verlag, pp 203-205, 2001.

10. Deller, J. R. Jr., Proakis, J. G., Hansen, J. H. L. “Discrete-time Processing of Speech Signals”, MacMillan Publishing Company:New York, 1993.

11. Smyth, P. "Clustering Sequences with Hidden Markov Models", Advances in Neural Information Processing Systems, Vol.9, pp 648-, The MIT Press, 1997.

12. Wahba, G. "Spline models for observational data". CBMS-NSF Regional Conference Series in Applied Mathematics Volume 59. SIAM, 1990. 